\title{
Wide-field spectroscopy and ELT science
}

\author{
Matthew Colless \\ Anglo-Australian Observatory, PO Box 296, Epping, NSW 1710, Australia \\ email: colless@aao.gov.au
}

\begin{abstract}
Wide-field spectroscopy, in its various forms, has much to contribute to ELT science, so care is needed in trade-offs between telescope size and field of view. Integral field spectroscopy over large areas at high spatial resolution, and especially multiple integral fields, will be essential tools. For wide-field surveys, next-generation multi-object spectrographs (MOS) on 8m-class telescopes will likely out-perform similar instruments on ELTs, due to the smaller fields of view of the current ELT designs. However, there may be $D^{4}$ gains for medium-resolution MOS if adaptive optics can provide enhanced 'seeing' of $\sim 0.1$ " over wide fields. New technologies such as $\mathrm{OH}$-suppression fibres offer revolutionary gains, so there is a difficult balance to be achieved in applying the latest technology and having instruments ready for ELT first light.
\end{abstract}

Keywords. instrumentation: spectrographs — instrumentation: miscellaneous - techniques: spectroscopic - telescopes

The meaning of the phrase 'wide-field spectroscopy' (WFS) depends on context: either wide in degrees (large physical scale at fixed sampling) or wide in pixels (fine sampling at fixed physical scale). Many types of astronomical observation can benefit wide-field spectroscopy in one or other of these strict senses of the term. However, the term is also closely associated with the common means of making best spectroscopic use of a wide field: most commonly multi-object spectroscopy (MOS), the usual point of a large physical field, and integral-field spectroscopy (IFS) the usual point of fine sampling of the field. Today, almost all 4m-class and 8m-class general-purpose telescopes have at least one wide-field spectrograph (MOS and/or IFS), and future instruments are likely to combine these modes in multiple integral field spectroscopy (MIFS).

A scan through the scientific cases that have been generated for ELTs reveals many goals that require, or would be far better off with, wide-field spectroscopy. One area I will focus on by way of example in this paper is cosmology and the early universe, where applications of WFS include: studies of first light objects and galaxy formation (IFS/MIFS); the emergence and evolution of large-scale structure and baryon tomography of the early universe (MOS); and deep spectroscopy of blank fields (IFS/MIFS).

As with so many other aspects of ELT science, the appropriate use of WFS is intimately linked to the issue of whether the targets are resolved (both in the sense of resolving a point source into an extended object and in the sense of resolving a single point source from a crowded field) and hence to the use of adaptive optics (AO). For cosmology and early-universe studies, the relevant scales are those of galaxies themselves and of galaxy clustering. For redshifts $z>0.5$, galaxy size does not depend on distance per se, though it evolves with redshift as galaxies assemble. Studies of galaxies over redshifts out to $z \sim 6$ show that their mean physical sizes are diminishing, from a few kpc at $z \sim 0$ to somewhat less than a kpc at $z \sim 6$ (Bouwens et al. 2004). This means that the most distant galaxies typically have sizes of around $0.1^{\prime \prime}$. However, there is likely to be structure on both smaller and larger scales, especially during the most active periods of galaxy assembly: the brightest star-forming components of proto-galaxies may be unresolvable individually, but may form relatively extended structures. Genuinely wide-field MOS (i.e. field of view 
Table 1. The applicability to various ELT science observations of multi-object spectroscopy (MOS), integral field spectroscopy (IFS) and deployable multi integral fields (MIF) is indicated by ticks and crosses (more ticks is better). The most appropriate AO mode, amongst natural seeing (NS), ground layer adaptive optics (GLAO), multi-object adaptive optics (MCAO) and multi-conjugate adaptive optics (MCAO), is indicated by shading.

\begin{tabular}{|l|c|c|c|c|}
\hline Science & FoV? & MOS? & IFS? & MIF? \\
\hline Resolved stellar populations & $>10^{\prime}$ & $\checkmark \checkmark$ & $\checkmark$ & $\mathbf{x}$ \\
\hline Massive stars beyond the Local Group & $\sim 1^{\prime}$ & $\checkmark$ & $\mathbf{x}$ & $\mathbf{x}$ \\
\hline Stellar kinematics archaeology & $>10^{\prime}$ & $\mathbf{x}$ & $\checkmark$ & $\checkmark \checkmark$ \\
\hline Young massive star clusters & $2^{\prime}$ & $\checkmark$ & $\boldsymbol{?}$ & $\mathbf{x}$ \\
\hline Astrophysics of massive black holes & $5^{\prime \prime}$ & $\mathbf{x}$ & $\checkmark \checkmark$ & $\mathbf{x}$ \\
\hline Cosmic expansion from primary distance indicators & $>10^{\prime}$ & $\checkmark$ & $\checkmark$ & $\mathbf{x}$ \\
\hline The highest redshift galaxies (z>10) & $>10^{\prime}$ & $\checkmark$ & $\checkmark$ & $\checkmark \checkmark$ \\
\hline Galaxies \& AGN at the end of re-ionisation (z=5-10) & $>10^{\prime}$ & $\checkmark$ & $\checkmark$ & $\checkmark \checkmark$ \\
\hline Astrophysics of high-redshift galaxies & $>10^{\prime}$ & $\checkmark$ & $\checkmark$ & $\checkmark \checkmark$ \\
\hline The assembly of galaxy haloes & $>10^{\prime}$ & $\checkmark$ & $\checkmark$ & $\checkmark \checkmark$ \\
\hline Baryon tomography of the cosmic web & $>10^{\prime}$ & $\checkmark \checkmark$ & $\times$ & $\checkmark$ \\
\hline
\end{tabular}

Adaptive optics mode NS GLAO MOAO MCAO

of order a degree) is also desirable to tackle the emergence of large-scale clustering in the galaxy distribution, since the bottom-up growth of structures is largely offset by the greater bias of targets at higher redshifts. Indeed, a complete picture of galaxy formation, linking star formation and large-scale clustering by tracking the baryonic matter between the reservoirs of hot gas, cold gas and stars, will require a combination of MOS and MIFS because of the very wide range of scales involved.

With these considerations in mind, the main issues for WFS on ELTs are: What is the role of AO for WFS on ELTs? What are the gains (and losses) for WFS as function of primary mirror diameter $(D)$ ? What are the scientific trade-offs between mirror diameter and field of view $(\mathrm{FoV})$ ? When are there $D^{2}$ gains and when are there $D^{4}$ gains? How does AO complexity trade off against FoV? What minimum threshold to capabilities is set by the competition from $8 \mathrm{~m}$ WFS? What innovations are likely to contribute to WFS instruments on ELTs? Given complex instruments and long lead-times, but also the high rate of technical innovation, when is the optimum time for freezing instrument designs?

In considering the role of AO in WFS on ELTs, one has to consider the various techniques in terms of both the level of correction they provide and the field of view over which they operate. For our purposes there are four generic types of AO - in order of increasing technical difficulty they are: natural seeing (NS) with no correction but of course the full FoV; ground layer adaptive optics (GLAO) with partial correction over a wide (if not full) FoV; multi-conjugate adaptive optics (MCAO) providing almost full correction over a wide field; and multi-object adaptive optics (MOAO) affording almost full correction in small regions over the full FoV. For 'small' ELTs (e.g. GMT), natural-seeing WFS is feasible and scientifically interesting, whereas for 'large' ELTS (e.g. OWL), partial or nearly full correction AO is the only practical option. Natural seeing and GLAO are most naturally linked with MOS, while MCAO is most naturally linked with large monolithic integral fields and MOAO with multiple small integral fields. Table 1 maps out the most 
Table 2. The scientific capabilities of ELTs as a function of telescope diameter, taking account of the likely smaller fields of view for larger ELTs and the consequent limitations on survey astronomy (from Watson, Hook \& Colless 2006).

\begin{tabular}{lcccc} 
Science Case & $20 \mathrm{~m}$ & $30 \mathrm{~m}$ & $60 \mathrm{~m}$ & $100 \mathrm{~m}$ \\
\hline Solar System & Y & Y & Y & Y \\
Exo-Planets (direct detection): Gas Giants & Y & Y & Y & Y \\
Exo-Planets (direct detection): Exo-Earths & N & N & Y? & Y \\
Proto-Planetary disks & Y & Y & Y & Y \\
Resolved Stellar Population: Local Group & N? & Y & Y & Y \\
Resolved Stellar Population: Virgo & N & N & N & Y \\
\hline Population surveys: Local Group & Y & Y & N & N \\
\hline Massive Black Holes & Y & Y & Y & Y \\
Star formation History of the Universe & Y & Y & Y & Y \\
Physics of Galaxies and Dark Matter, $z=1-5$ & Y & Y & Y & Y \\
\hline Large-Scale Structure and Galaxy Formation & Y & Y & N & N \\
\hline Dark Energy & Y & Y & Y & Y \\
High-z Universe: Sources of re-ionisation & $?$ & $?$ & Y & Y \\
High-z Universe: $R=10^{4}$ on GRB at $z>10$ & N & N & Y & Y \\
The Unexpected & Y & Y & Y & Y
\end{tabular}

powerful combinations of WFS and AO techniques for a range of ELT science related to stellar populations, cosmology and the early universe.

While the relations between AO modes and WFS modes are reasonably straightforward, the same is not true of the trade-off between telescope diameter and WFS field of view. The desirability of larger primary mirrors is of course the raison d'être for ELTs. However, in practice it seems that increasing the primary mirror diameter comes at the cost of decreasing the field of view. While most of the $8 \mathrm{~m}$-class telescopes in the world have FoV approaching $30^{\prime}$ (and Subaru is considering an upgrade to a $1.5^{\circ} \mathrm{FoV}$ ), current designs for ELTs appear to be targeting smaller FoV: $20-25^{\prime}$ on the $20 \mathrm{~m}$ GMT, $20^{\prime}$ on the $30 \mathrm{~m}$ TMT and $10^{\prime}$ on the $100 \mathrm{~m}$ OWL. For survey astronomy, where the relevant figure of merit is usually the product of aperture and solid angle, $A \Omega \propto D^{2} \mathrm{FoV}^{2}$, these smaller FoV significantly offset the aperture gains of ELTs. For WFS, once the aperture is large enough to make a given observation viable (in terms of available telescope time, spatial resolution and reduced systematic errors), then for background-limited observations there is no gain at all from larger apertures if the field of view decreases with diameter as FoV $\propto D^{-1}$. Table 2 offers a slightly extended version of the table of science capabilities versus telescope size found in the European ELT science case that emphasizes the lack of gains from larger apertures beyond a certain point for survey science.

WFS on $8 \mathrm{~m}$-class telescopes is evolving rapidly. Future WFS instruments planned or being built for $8 \mathrm{~m}$ telescopes include: (i) FMOS on Subaru, a near-infrared, $R \sim 800$, sky-suppression MOS with 400 fibres and a $30^{\prime} \mathrm{FoV}$; (ii) MUSE on the VLT, an optical, $R \sim$ 2000-4000 IFS with $10^{5}$ pixels, utilizing AO to cover a $1^{\prime} \times 1^{\prime}$ FoV at $0.2^{\prime \prime}$ per pixel; and (iii) WFMOS for Subaru/Gemini, an optical, $R \sim 3000-40000$ MOS with 4500 fibres covering a $1.5^{\circ} \mathrm{FoV}$. These instruments provide a high minimum threshold of performance for any wide-field spectrograph on an ELT — e.g. WFMOS on Subaru will have higher $A \Omega$ (and so be more efficient for surveys) than any $30 \mathrm{~m}$ WFS with FoV $<24^{\prime}$; it will also have at least 5 years head start, and will likely be available for more nights per year because of lower time pressure on $8 \mathrm{~m}$ telescopes than ELTs.

So is WFS on ELTs worthwhile? Although it may not be a primary driver for ELTs, WFS will certainly be a valuable facility on ELTs, especially if ELT fields of view are at least comparable to those of $8 \mathrm{~m}$-class telescopes. In addition, WFS could in principle 
Table 3. The variation in the speed of observations with telescope diameter $D$ and spatial resolution $r$ for different noise and AO regimes and for resolved and unresolved sources (adapted from Taylor, Britton \& Jones 2005).

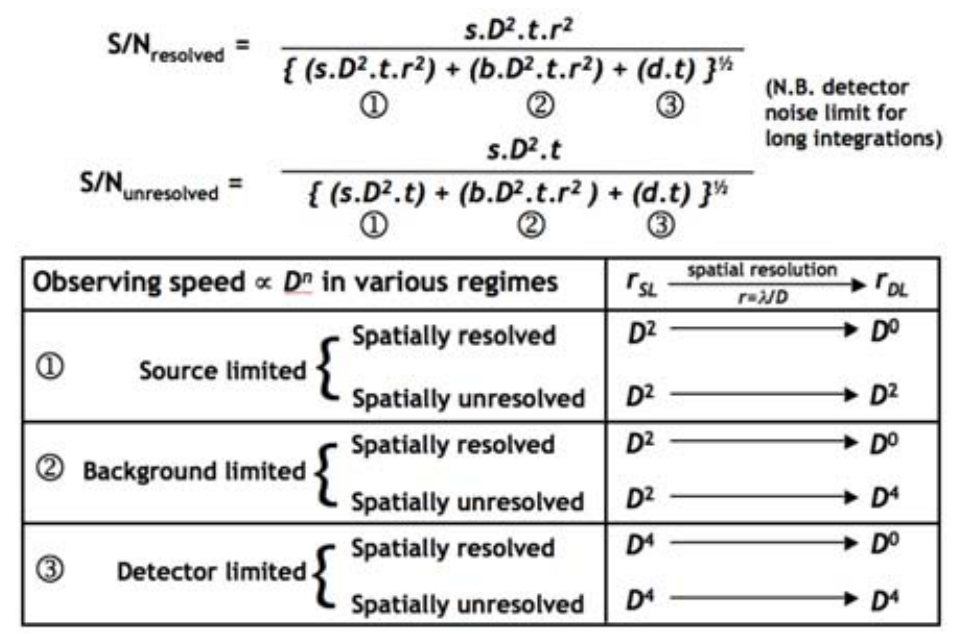

gain enormously from larger telescopes, even if FoV decreases with increasing diameter, for scientific applications in a regime where the gain with aperture in the speed of observations grows as $D^{4}$ rather than $D^{2}$, as has been emphasized recently by Taylor, Britton \& Jones (2005). Table 3 shows how speed of observation (i.e. inverse integration time) varies with telescope diameter $D$ and effective spatial resolution $r$ depending on the noise regime (source-, background- or detector-limited), the spatial resolution regime (seeing-limited or diffraction-limited) and whether the source is resolved or unresolved.

The table shows that there is a $D^{4}$ gain in speed in either of two cases: (i) if the observation is sky- or detector-noise limited and the source is unresolved at the diffraction limit, or (ii) if the observation is detector-noise limited and at the 'seeing' limit (which might be enhanced by AO). The first case might apply to medium- $R$ spectra of individual stars in nearby galaxies or high- $R$ spectra of absorption lines in QSO spectra. The second case might in principle be relevant to enhanced-seeing MOS observations on an ELT.

Taylor, Britton \& Jones (2005) show that if the 'seeing' limit is smaller than a certain critical spatial resolution (corresponding to the detector area over which the sky noise equals the detector noise, and therefore dependent on the spectral resolution), then $D^{4}$ gains can be made. They have investigated the combinations of spectral and spatial resolution that yield this regime on a $30 \mathrm{~m}$ telescope, making reasonable assumptions for spectrograph throughput and the performance of optical and near-infrared detectors. They find that $D^{4}$ gains are realized if $R>4000$ and the AO-enhanced seeing limit is $<0.1^{\prime \prime}$. This regime is of scientific interest, since high-redshift galaxies are typically about this size and $R \sim 4000$ would be well matched to their expected internal motions of $<100 \mathrm{~km} \mathrm{~s}^{-1}$. However, whether AO techniques can deliver this level of enhanced seeing over sizeable fields is as yet unclear.

Finally, the future of WFS cannot neglect the remarkable innovations that are occurring in fibre technology, including: new fibre materials leading to high-performance fibres for the UV and mid-infrared; photonic crystal (microstructured) fibres providing large numerical apertures for ELTs' fast $(<f / 2)$ beams and aperture transforms via graded cores; concentric fibres for simultaneous sky subtraction; and aperiodic fibre Bragg gratings (AFBGs) that suppress the $\mathrm{OH}$ sky-lines without throughput losses or scattered 


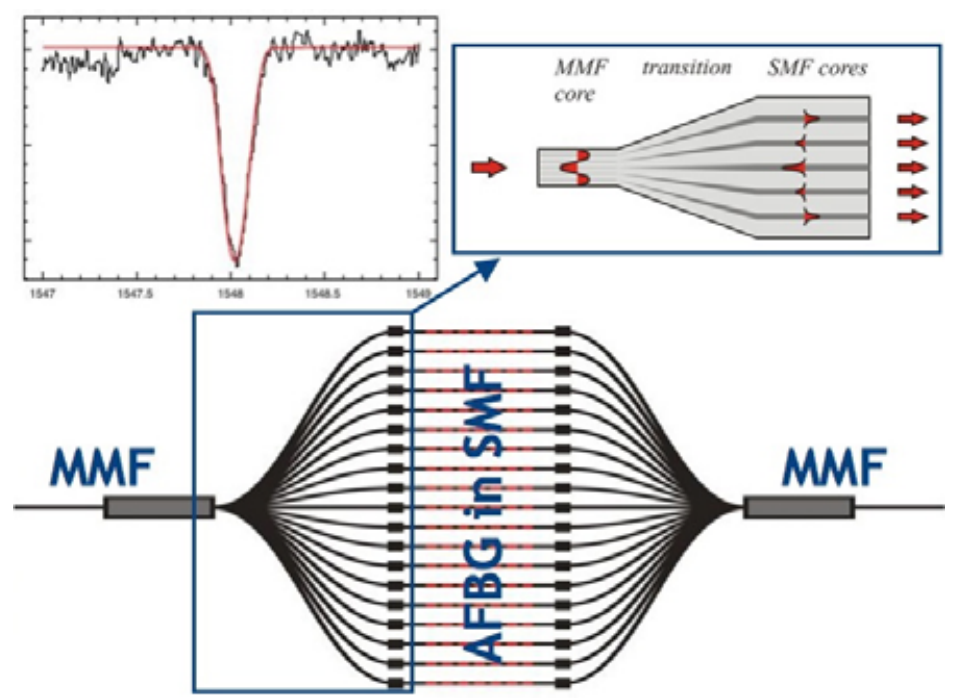

Figure 1. Bottom: schematic of the 'chinese lantern' arrangement for feeding light from multi-mode fibres (MMF) through multiple single-mode fibres (SMF) with aperiodic fibre Bragg gratings (AFBG). Top right: blow-up of the MMF-SMF transition. Top left: the performance of this arrangement in removing a single sky line (predicted-smooth curve; measured-noisy curve).

light. AFBGs will lead to a revolution in near-infrared spectroscopy, allowing observations to reach the near-infrared sky continuum background by almost perfect suppression of $\mathrm{OH}$ sky lines. Figure 1 shows the latest development of this technology at the AngloAustralian Observatory, in which aperture transformations are used to couple multi-mode and single-mode fibres to combine the large numerical aperture of MMFs with AFBGs in SMFs, permitting $\mathrm{OH}$-suppression in natural-seeing spectrographs. Details can be found in Bland-Hawthorn, Englund \& Edvell (2004) and Bland-Hawthorn (2006).

\section{Acknowledgements}

I acknowledge stimulating discussions with the Australian ELT Working Group.

\section{References}

Bland-Hawthorn, J. 2006, AAO Newsletter 108, 4

Bland-Hawthorn, J., Englund, M.A. \& Edvell G. 2004, Optics Express 12, 5902

Bouwens, R.J., Illingworth, G.D., Blakeslee, J.P., Broadhurst, T.J. \& Franx, M. 2004, ApJ 611, L1

Taylor, K., Britton, M. \& Jones, D.A. 2005, in: T. Herbst (ed.), Instrumentation for Extremely Large Telescopes, (Ringberg Workshop: http://www.mpia-hd.mpg.de/ELT)

Watson, F.G., Hook, I.M. \& Colless, M.M. 2006, in: J.W. Mason (ed.), Astrophysics Update 2 (Berlin: Springer), chap. 11, in press 